\title{
Effect of Smartphone-Based Lifestyle Coaching App on Community-Dwelling Population With Moderate Metabolic Abnormalities: Randomized Controlled Trial
}

So Mi Jemma Cho ${ }^{1 *}$, BA; Jung Hyun Lee ${ }^{2 *}$, MD; Jee-Seon Shim ${ }^{3 *}$, PhD; Hyungseon Yeom², MD; Su Jin Lee ${ }^{4}$, MD; Yong Woo Jeon ${ }^{1}$, MD; Hyeon Chang Kim ${ }^{1,3}$, MD, PhD, FAHA

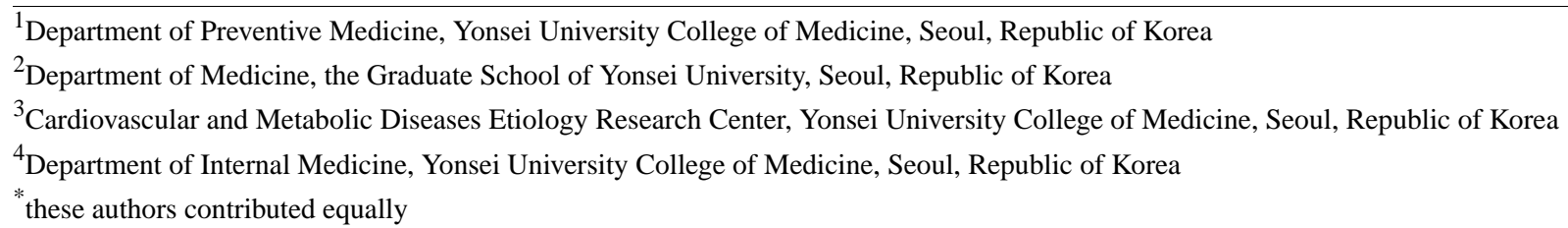

\section{Corresponding Author:}

Hyeon Chang Kim, MD, PhD, FAHA

Department of Preventive Medicine

Yonsei University College of Medicine

50-1 Yonsei-ro, Seodaemun-gu

Seoul, 03722

Republic of Korea

Phone: 82222281873

Email: hckim@yuhs.ac

\section{Abstract}

Background: Metabolic disorders are established precursors to cardiovascular diseases, yet they can be readily prevented with sustained lifestyle modifications.

Objective: We assessed the effectiveness of a smartphone-based weight management app on metabolic parameters in adults at high-risk, yet without physician diagnosis nor pharmacological treatment for metabolic syndrome, in a community setting.

Methods: In this 3-arm parallel-group, single-blind, randomized controlled trial, we recruited participants aged 30 to 59 years with at least 2 conditions defined by the Third Report of the National Cholesterol Education Program expert panel (abdominal obesity, high blood pressure, high triglycerides, low high-density lipoprotein cholesterol, and high fasting glucose level). Participants were randomly assigned (1:1:1) by block randomization to either the nonuser group (control), the app-based diet and exercise self-logging group (app only), or the app-based self-logging and personalized coaching from professional dieticians and exercise coordinators group (app with personalized coaching). Assessments were performed at baseline, week 6, week 12, and week 24. The primary outcome was change in systolic blood pressure (between baseline and follow-up assessments). Secondary outcomes were changes in diastolic blood pressure, body weight, body fat mass, waist circumference, homeostatic model of assessment of insulin resistance, triglyceride level, and high-density lipoprotein cholesterol level between baseline and follow-up assessments. Analysis was performed using intention-to-treat.

Results: Between October 28, 2017 and May 28, 2018, 160 participants participated in the baseline screening examination. Participants $(129 / 160,80.6 \%)$ who satisfied the eligibility criteria were assigned to control $(n=41)$, app only $(n=45)$, or app with personalized coaching $(n=43)$ group. In each group, systolic blood pressure showed decreasing trends from baseline (control: mean -10.95 , SD $2.09 \mathrm{mmHg}$; app only: mean -7.29 , SD $1.83 \mathrm{mmHg}$; app with personalized coaching: mean -7.19 , SD 1.66 $\mathrm{mmHg}$ ), yet without significant difference among the groups (app only: $P=.19$; app with personalized coaching: $P=.16$ ). Instead, those in the app with personalized coaching group had greater body weight reductions (control: mean $-0.12, \mathrm{SD} 0.30 \mathrm{~kg}$; app only: mean -0.35 , SD $0.36 \mathrm{~kg}, P=.67$; app with personalized coaching: mean -0.96, SD $0.37 \mathrm{~kg} ; P=.08$ ), specifically by body fat mass reduction (control: mean -0.13 , SD $0.34 \mathrm{~kg}$; app only: mean $-0.64, \mathrm{SD} 0.38 \mathrm{~kg}, P=.22$; app with personalized coaching: mean -0.79 , SD $0.38 \mathrm{~kg} ; P=.08)$. 
Conclusions: Simultaneous diet and exercise self-logging and persistent lifestyle modification coaching were ineffective in lowering systolic blood pressure but effective in losing weight and reducing body fat mass. These results warrant future implementation studies of similar models of care on a broader scale in the context of primary prevention.

Trial Registration: ClinicalTrials.gov NCT03300271; http://clinicaltrials.gov/ct2/show/NCT03300271

(J Med Internet Res 2020;22(10):e17435) doi: 10.2196/17435

\section{KEYWORDS}

metabolic health; health behavior; lifestyle modification; mobile health

\section{Introduction}

Metabolic disorders are established precursors to cardiovascular disease [1]. Previous studies have demonstrated that even at subclinical stages, persons with elevated blood pressure, blood glucose level, cholesterol level, and adiposity are at significantly higher risk of adverse cardiometabolic outcomes [2]. Despite recent health care policy changes that have expanded healthy lifestyle advocacy initiatives, a considerable proportion do not achieve the guideline-recommended metabolic profile [3-5]. Therefore, timely and persistent management of metabolic abnormalities are crucial in preventing adverse health outcomes at an individual level and conserving substantial health care costs at a national level.

Alongside the advent of new medicines, technological innovations have aided in easing accessibility to and enriching the quality of health care. They have eliminated practical barriers, thereby allowing the distribution and improvement of health care via nonconventional routes at unprecedented speeds [6]. Several features include the transmission of medical records, social media forums for open discussion, web-based interactive education programs, higher precision diagnostics, real-time status tracking, digitalized clinics (ie, telemonitoring), and prescription dispensation $[7,8]$.

In particular, the ubiquity of mobile phone technology has incentivized industries to create smartphone-based apps for health monitoring [9]. Previous trials [10,11] have evaluated the efficacy and effectiveness of such tools in the context of secondary prevention. For example, among patients receiving cardiac rehabilitation after hospitalization for myocardial infarction, daily text message reminders led to greater medication adherence and exercise capacity compared with patients receiving usual care [10]. The utility extends to general populations; a Finnish trial [11] demonstrated both short- and long-term weight loss among people who were overweight and who logged weight daily and received dietary management instructions over a period of 1 year. By collecting data in real time, these mobile-based apps enable researchers to assess multiple behaviors and to prompt change at low cost and with high ease.

Nonetheless, there are important limitations in the current literature. Previous studies [12-14] have primarily recruited clinic patients who were already using or were exceptionally motivated to use health management tools. Methodologically, many studies [7] estimated the effect of these mobile interventions based on per protocol analysis. It has been established that physical activity and diet affect blood pressure level not only in hypertension patients but also in people with prehypertension or elevated blood pressure [15]. Recent blood pressure guidelines [15,16] emphasized early lifestyle modification for people whose blood pressure is above the normal range. However, the effects of smartphone-based apps in lowering blood pressure have not been properly assessed in people whose blood pressure is above the normal range in real-world settings.

In this context, the objective of the study was to evaluate the longitudinal effect of smartphone-based health care app on metabolic parameters in a sample of the general population with moderate metabolic abnormalities yet without clinical diagnosis nor pharmacological treatment. We hypothesized that the participants receiving both real-time personalized coaching and self-logging diet and physical activity would yield greater improvements in blood pressure and other metabolic parameters than those who were only self-logging or who did not use the app.

\section{Methods}

\section{Study Design}

The study was designed as a single-blind 3-arm parallel-design randomized controlled trial delivering a 6-month primary prevention program via mobile app to a population with moderate metabolic abnormalities with neither diagnosis nor treatment for metabolic disorders. The main objective was to evaluate the effectiveness of health management app on metabolic parameters over 3 follow-up examinations. Community-dwelling adults residing in Seoul and nearby capital regions were recruited. The study protocol was approved by the institutional review boards of Severance Hospital and Yonsei University Health System (4-2017-0666), and the protocol of the study is registered at ClinicalTrials.gov (NCT03300271).

\section{Participants}

Candidate participants were identified based on objectively measured metabolic profiles from a previous Cardiovascular and Metabolic Diseases Etiology Research Center (CMERC) observational cohort study [17]. Briefly, the CMERC study [17] aimed to identify novel risk factors and to investigate the distribution and effects of known cardiovascular and metabolic diseases risk factors.

We recruited smartphone users aged 30-59 years with at least 2 metabolic abnormalities defined by the modified version of the Third Report of the National Cholesterol Education Program expert panel on detection, evaluation, and treatment of high blood cholesterol in adults [18] using the criteria for Asian 
populations: waist circumference (male: $\geq 90 \mathrm{~cm}$; female: $\geq 80$ $\mathrm{cm}$ ), blood pressure (systolic: $\geq 135 \mathrm{mmHg}$; diastolic: $\geq 85$ $\mathrm{mmHg}$ ), triglyceride $\geq 150 \mathrm{mg} / \mathrm{dL}$, high-density lipoprotein (HDL) cholesterol (male: $<40 \mathrm{mg} / \mathrm{dL}$; female: $<50 \mathrm{mg} / \mathrm{dL}$ ), and fasting glucose level $\geq 100 \mathrm{mg} / \mathrm{dL}$. Exclusion criteria included users of smartphone-based health care apps for lifestyle modification, individuals with a previous diagnosis of cardiovascular disease, malignant cancer, or metabolic syndrome, individuals who were taking antihypertensives, lipid- or glucose-lowering drugs, and women who were pregnant or breastfeeding at the time of the study.

Among 3625 CMERC cohort participants [17], 546 people qualified. We contacted these individuals via telephone and mail for recruitment. Of the $160(29.1 \%)$ who expressed affirmative for screening test, a total of 129 participants (23.6\%) attended the baseline examination (Figure 1).

Participants were asked to give their written consent without knowledge of the intervention assignments.

Figure 1. Flow diagram of the study participants. CMERC: Cardiovascular and Metabolic Diseases Etiology Research Center.

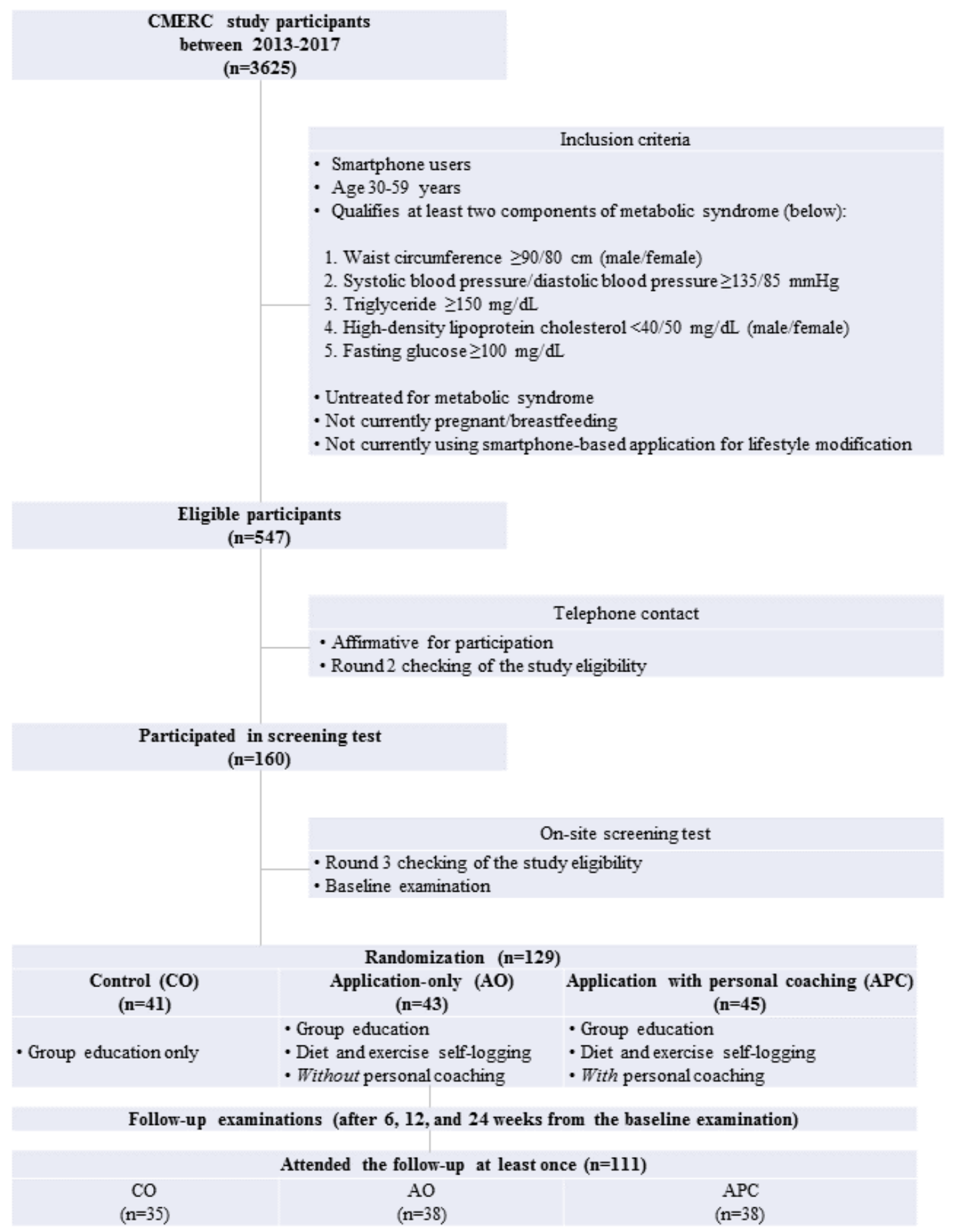




\section{Randomization and Masking}

We randomly allocated participants into 1 of the 3 intervention arms via sex- and age-stratified block randomization. Specifically, we set the block size equal to 6 from sex (male or female) by age group in decile (30, 40, and 50) using $\mathrm{R}$ (version 3.4.4; R Foundation for Statistical Computing). We adopted a single-blind approach; thus, the effectiveness would be assessed by masked researchers unaware of the randomization results. The allocation concealment was achieved via individualized texting, which instructed all participants to adhere to their respective intervention, to avoid using other health management apps, and to refrain from sharing of their intervention instructions with each other for the entire study duration. To minimize crossover or contamination, we (1) retrospectively ensured no unallocated feature had been installed to the app only group from the app's user-specific metadata and (2) asked the participants whether they had engaged in other health-related trials, programs, or apps at every follow-up assessment.

\section{Procedures}

At the baseline assessment, all participants received on-site education that entailed information on metabolic syndrome and preventive strategies, including validated exercise regimens and nutritious cooking recipes. Then, we randomly allocated participants into 1 of the 3 intervention arms: the control group (control; $n=41$ ) received only the aforementioned baseline education and was asked to refrain from concurrently engaging in any smartphone-based lifestyle modification app or programs; the app only (app only; $n=45$ ) and the app with personalized coaching (app with personalized coaching; $n=43$ ) groups also received the baseline education and were additionally asked to use a smartphone-based weight management app called Noom (Noom Inc). Specifically, Noom allows users to log details regarding daily food intake and physical activities. For instance, a user can record a specific menu item (ie, fastfood chain M's cheeseburger), consumed portion (ie, single serving, half of cheeseburger), and time of consumption (ie, breakfast, 8 AM). From Noom's nutrition metadata, the app readily calculates total calories and macronutrients consumed for each log entry. Likewise, a wide array of physical activities (ie, indoor treadmill walking) are available for selection from the predefined list. After entering the intensity (ie, pace) and duration (ie, 60 minutes) of physical activity, the app yields energy expenditure in kilocalories, accounting for the user's sex, age, height, and weight. As an additional feature, it delivers structured health-related curricula and personalized feedback from certified exercise regimen coordinators and clinical dieticians based on their reviews of the user's logs (Figure 2).

Figure 2. Example of personalized coaching.

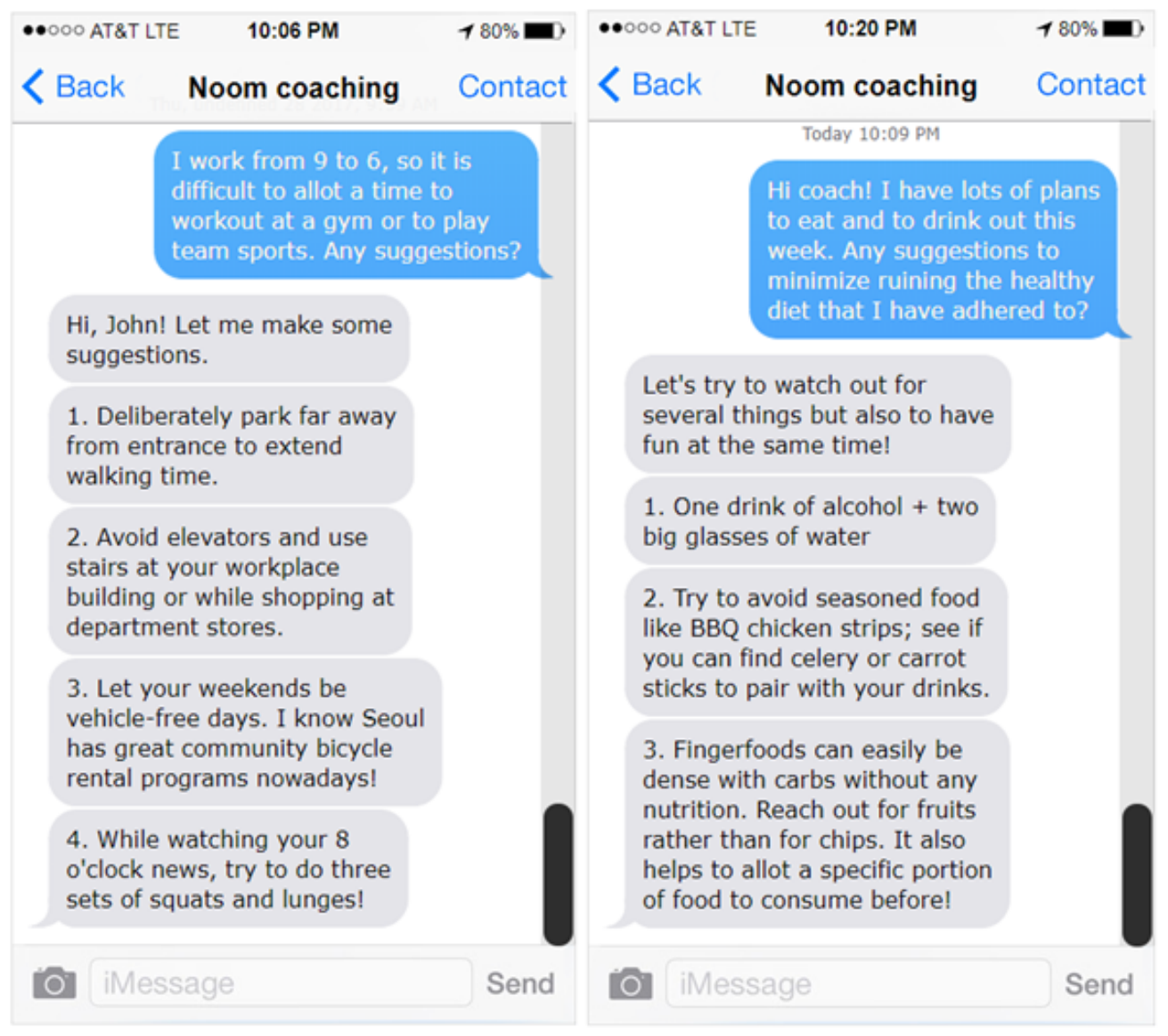

The default frequency of personalized coaching was set to 3 times per week; however, the actual frequency varied depending on the user's participation rate. Only the app with personalized coaching group received the personalized coaching. All users' 
activation statuses were evaluated based on the number of weight, meal, and physical activity logs. Weekly, we defined each user as active if the user had recorded at least 1 aforementioned parameter.

The universal baseline examination included anthropometric measurements, blood tests, and face-to-face interviews on demographics, disease history, and health behavior. Then, participants received text messages notifying them of the study initiation. The app only and app with personalized coaching groups were given additional instructions regarding the Noom app installation procedures; each user received a unique identification code.

\section{Outcomes}

The primary outcome was change in systolic blood pressure between the baseline and follow-up at 6,12 , and 24 weeks. The secondary outcomes were changes in diastolic blood pressure, body weight, body fat mass, waist circumference, homeostatic model of assessment of insulin resistance (HOMA-IR), and lipid profile (triglyceride and HDL cholesterol) between the baseline and follow-up at 6, 12, and 24 weeks.

Anthropometric measurements were performed with strict adherence to standardized protocols and using calibrated equipment. Blood pressure was consecutively measured using both single- and double-arm automated oscillometric devices (HEM-7080, Omron Health; WatchBP Office Central, Microlife) at a single sitting. The mean of second and third measurements were used for analysis. Participants underwent blood tests after overnight fasting for a minimum of 8 hours. Fasting plasma glucose and insulin levels were assessed using colorimetry method (ADVIA1800 Auto Analyzer, Siemens Medical Solutions). HOMA-IR was calculated as the product of fasting glucose and insulin levels divided by 405 in $\mathrm{mg} / \mathrm{dL}$. Weight was measured to the nearest $0.1 \mathrm{~kg}$ on a digital scale (DB-150, CAS). To minimize measurement variability, a zero-point adjustment was routinely conducted using weight blocks (20, 40 , and $60 \mathrm{~kg}$ ). Bioelectrical impedance analysis delineated body composition (BSM-330, INBODY). Waist circumference was measured to the nearest $0.1 \mathrm{~cm}$ using a plastic tape (SECA 201, SECA), while maintaining the level of the measuring tape.

Physical activity was assessed by the Korean version of the International Physical Activity Questionnaire [19]. Using validated alcohol consumption and cigarette smoking ratings, participants were divided into non-, previous-, and current categories for alcohol consumption and smoking.

\section{Statistical Analysis}

The target sample size was 150 participants, chosen to provide precise estimates of the intended effect of mobile app usage. Specifically, the sample size calculation was conducted based on the expected difference in systolic blood pressure after 24 weeks across the 3 intervention arms [20]. We assumed a statistical power of $80 \%$ and a significance level of $P<.05$. Based on previous literature [20,21], we expected a mean systolic blood pressure difference of $6 \mathrm{mmHg}$ with a standard deviation of $10 \mathrm{mmHg}$ after 24 weeks. When considering $10 \%$ attrition rate and 3-arm design, the study required a minimum recruitment of 150 participants.

We employed analysis of variance to assess differences in demographic and health-related behavior. Then, we compared the extent of changes in each metabolic parameter across participants randomly assigned to control versus app only and app with personalized coaching groups, separately, using an intention-to-treat approach; all participants who participated in at least 1 of the 3 follow-up assessment were included in the analysis. We used independent $t$ tests to evaluate the primary and secondary outcomes at each time point. To account for repeated measurements over multiple follow-ups, we employed a linear mixed model to determine the effect of mobile health care apps on the prespecified outcomes. Specifically, the unstructured linear mixed model incorporates time and group $\times$ time interaction terms to assume no homogeneity across the 3 groups at the baseline. From the random intercept model, the estimated beta coefficient of the group $\times$ time interaction term was regarded as the effect of the app usage. The changes are presented as estimated beta coefficient $(\beta)$ and standard error (SE). All statistical tests were 2-sided and the statistical significance was set at a $P<.05$. All analyses were performed using $\mathrm{R}$ and SAS (version 9.4; SAS Institute Inc).

All data were collected, registered, and managed on ClinicalTrials.gov (NCT03300271). Data were deidentified and accessible only by designated researchers. All researchers strictly adhered to data security protocols. For unbiased data monitoring and trial safety overseeing, the research director delegated Dae Ryong Kang, a professor of biomedical data science at Yonsei University, Wonju College of Medicine, Wonju, Korea, who remained independent of the research execution.

\section{Results}

\section{Participants}

All participants were enrolled on October 28, 2017, and the last participant completed the week 24 follow-up on June 2, 2018. Of the 129 enrolled individuals, 41 (32\%) were randomly assigned to the control group, $45(35 \%)$ to the app only group, and $43(33 \%)$ to the app with personalized coaching group (Figure 1). In the end, 111 participants attended at least 1 follow-up examination (week 6: 107; week 12: 100; week 24: $105)$, yielding a $14.0 \%$ attrition rate overall.

\section{Baseline Characteristics}

Table 1 shows the general characteristics of the study participants at the baseline screening. Overall, the participants were similarly distributed in terms of age, metabolic parameters, and health behaviors across the 3 groups. At baseline, systolic blood pressure was comparable across the 3 groups (control: mean $131.8 \mathrm{mmHg}$; app only: mean $130.8 \mathrm{mmHg}$; app with personalized coaching: mean $133.3 \mathrm{mmHg}$ ). Such comparability across the groups ensured the baseline differences did not affect the changes in metabolic parameter over the study period. 
Table 1. General characteristics of the study participants at the baseline screening.

\begin{tabular}{|c|c|c|c|}
\hline Variables & Control $(n=41)$ & App only $(n=45)$ & $\begin{array}{l}\text { App+personalized coaching } \\
(n=43)\end{array}$ \\
\hline Age (years), mean (SD) & $49.5(7.9)$ & $49.2(7.5)$ & $48.9(7.8)$ \\
\hline \multicolumn{4}{|l|}{ Sex, n (\%) } \\
\hline Male & $19(46.3)$ & $23(51.1)$ & $21(48.8)$ \\
\hline Female & $22(53.7)$ & $22(49.9)$ & $22(51.2)$ \\
\hline Number of metabolic abnormalities, mean (SD) & $2.9(0.8)$ & $2.9(0.9)$ & $2.8(0.9)$ \\
\hline Systolic blood pressure (mmHg), mean (SD) & $131.8(15.8)$ & $130.8(15.2)$ & $133.3(14.9)$ \\
\hline Diastolic blood pressure (mmHg), mean (SD) & $87.4(9.9)$ & $86.6(10.7)$ & $89.0(11.7)$ \\
\hline Height (cm), mean (SD) & $166.4(8.8)$ & $165.3(10.4)$ & $164.0(8.9)$ \\
\hline Weight (kg, mean (SD) & $71.8(13.2)$ & $72.6(12.4)$ & $71.9(11.6)$ \\
\hline Body mass index $\left(\mathrm{kg} / \mathrm{m}^{2}\right)$, mean (SD) & $25.8(2.8)$ & $26.5(3.2)$ & $26.6(2.9)$ \\
\hline Waist circumference $(\mathrm{cm})$, mean $(\mathrm{SD})$ & $89.1(8.2)$ & $90.8(8.2)$ & $90.4(7.0)$ \\
\hline Percent body fat, mean (SD) & $30.3(6.8)$ & $31.9(7.1)$ & $31.6(5.7)$ \\
\hline Fat mass $(\mathrm{kg})$, mean $(\mathrm{SD})$ & $21.5(5.2)$ & $23.1(6.4)$ & $22.5(4.5)$ \\
\hline Skeletal muscle mass $(\mathrm{kg})$, mean $(\mathrm{SD})$ & $28.1(7.1)$ & $27.6(6.2)$ & $27.5(6.1)$ \\
\hline Total cholesterol (mg/dL), mean (SD) & $212.9(31.6)$ & $197.4(34.1)$ & $205.1(31.8)$ \\
\hline Triglyceride (mg/dL), mean (SD) & $212.9(138.0)$ & $176.6(107.7)$ & $169.2(72.7)$ \\
\hline HDL $^{\mathrm{a}}$ cholesterol (mg/dL), mean (SD) & $46.1(8.4)$ & $47.1(11.2)$ & $46.3(10.5)$ \\
\hline $\mathrm{LDL}^{\mathrm{b}}$ cholesterol (mg/dL), mean (SD) & $139.1(28.8)$ & $127.5(35.0)$ & $137.1(30.7)$ \\
\hline Hemoglobin $\mathrm{A}_{1 \mathrm{c}}(\%)$, mean $(\mathrm{SD})$ & $5.9(0.7)$ & $6.1(1.2)$ & $5.8(0.7)$ \\
\hline HOMA-IR ${ }^{\mathrm{c}}$, mean (SD) & $2.8(1.2)$ & $3.0(1.5)$ & $2.9(1.5)$ \\
\hline Hypertension, mean (SD) & $2(4.9)$ & $2(4.4)$ & $1(2.3)$ \\
\hline Diabetes, mean (SD) & $0(0.0)$ & $0(0.0)$ & $0(0.0)$ \\
\hline Dyslipidemia, mean (SD) & $3(7.3)$ & $1(2.2)$ & $1(2.3)$ \\
\hline \multicolumn{4}{|l|}{ Smoking status, n (\%) } \\
\hline Never & $19(46.3)$ & $28(62.2)$ & $25(58.1)$ \\
\hline Former & $8(19.5)$ & $11(24.4)$ & $11(25.6)$ \\
\hline Current & $14(34.2)$ & $6(13.3)$ & $7(16.3)$ \\
\hline \multicolumn{4}{|l|}{ Alcohol consumption, n (\%) } \\
\hline Never & $5(12.2)$ & $8(17.8)$ & $5(11.6)$ \\
\hline Former & $2(4.9)$ & $0(0.0)$ & $2(4.7)$ \\
\hline Current & $34(82.9)$ & $37(82.2)$ & $36(83.7)$ \\
\hline Alcohol consumption (g/day), mean (SD) & $22.1(38.8)$ & $10.4(15.5)$ & $11.5(18.1)$ \\
\hline \multicolumn{4}{|l|}{ MVPA $^{d}$ engagement, n (\%) } \\
\hline Yes & $2(4.9)$ & $8(17.8)$ & $9(20.9)$ \\
\hline No & $39(95.1)$ & $37(82.2)$ & $34(79.1)$ \\
\hline Sedentary time (hours/day), mean (SD) & $7.2(2.7)$ & $6.6(2.8)$ & $7.1(3.9)$ \\
\hline
\end{tabular}

${ }^{\text {a}}$ HDL: high-density lipoprotein.

${ }^{\mathrm{b}}$ LDL: low-density lipoprotein. 
${ }^{\mathrm{c}}$ HOMA-IR: homeostatic model assessment of insulin resistance.

${ }^{\mathrm{d}}$ MVPA: moderate-vigorous physical activity.

To ensure homogeneity between those who attended and those who did not attend follow-up at least once, we have compared the baseline characteristics as illustrated in Multimedia Appendix 1. In regard to age, sex, anthropometry, and glycemic and lipid profiles, no statistically significant differences (age: $P=.10$; sex: $P=.09$; systolic blood pressure: $P=.36$; diastolic blood pressure: $P=.93$; BMI: $P=.98$; total cholesterol: $P=.95$; fasting glucose level: $P=.25$ ) were detected between the 2 groups. The only notable difference was the proportion of current smokers; those who did not attend any follow-up examinations had a higher proportion of current smokers than those who attended at least once $(44.4 \%$ versus $17.1 \% ; P=.04$; Multimedia Appendix 2).

\section{Comparisons at Each Time Point}

All 3 groups showed varying amounts of systolic blood pressure reduction overall. In reference to the baseline examination, the control and app only groups showed the most dramatic reduction at week 24 (control: mean -10.95, SD $11.98 \mathrm{mmHg}$; app only: mean -7.19 , SD $9.98 \mathrm{mmHg}$ ), whereas the app with personalized coaching group showed the most dramatic systolic blood pressure reduction at week 12 (mean -7.82, SD $11.98 \mathrm{mmHg}$ ). However, compared to the control group, neither the app only nor the app with personalized coaching group had significantly different systolic blood pressure change at any follow-up examination (Figure 3).

For secondary outcomes, participants in the app with personalized coaching group generally lost more body weight (mean $-0.96 \mathrm{~kg}$ versus $-0.12 \mathrm{~kg}$; Figure 4 ), lost more body fat mass (mean $-0.79 \mathrm{~kg}$ versus $0.13 \mathrm{~kg}$; Figure 5), and had smaller waist circumferences (mean $-1.86 \mathrm{~cm}$ versus $-0.08 \mathrm{~cm}$; Multimedia Appendix 2) by the end of the study period. At week 24, diastolic blood pressure ( $P=.42$; Multimedia Appendix 3 ) and other glycemic (fasting glucose level: $P=.99$; HOMA-IR: $P=.63$; Multimedia Appendix 4 and Multimedia Appendix 5, respectively), and lipid indices (triglyceride level: $P=.93$; HDL cholesterol level: $P=.46$; Multimedia Appendix 6 and Multimedia Appendix 7, respectively) had not changed significantly.

Figure 3. Systolic blood pressure changes from the baseline across the 3 groups at each time point. AO: app-only; APC: app with personalized coaching; CO: control, SBP: systolic blood pressure.

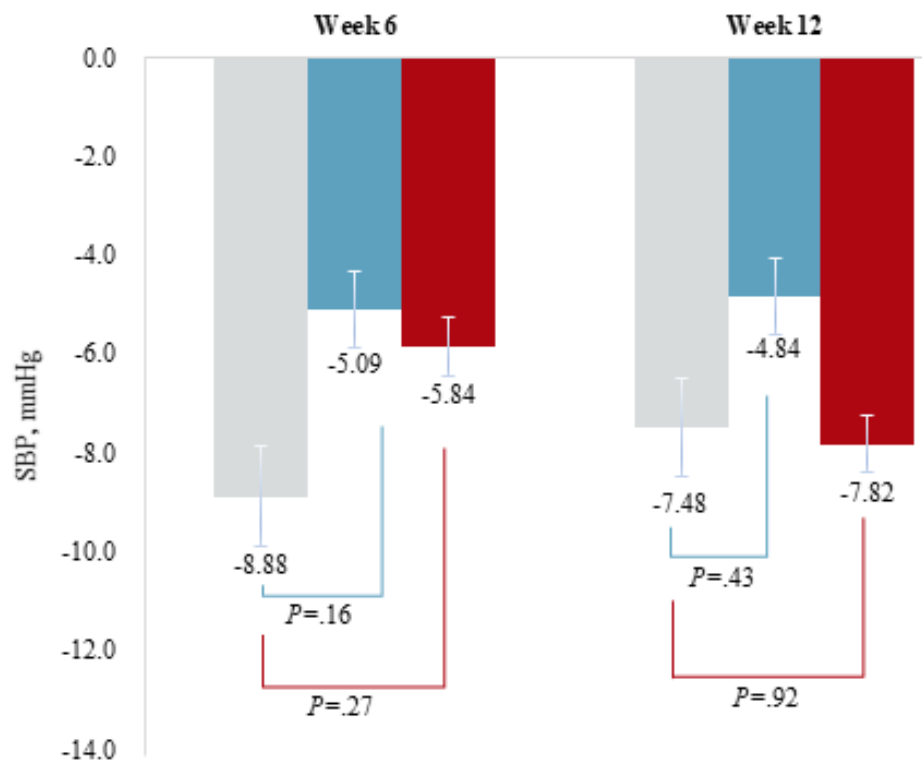

$\square \mathrm{CO}=\mathrm{AO}=\mathrm{APC}$
Week 24

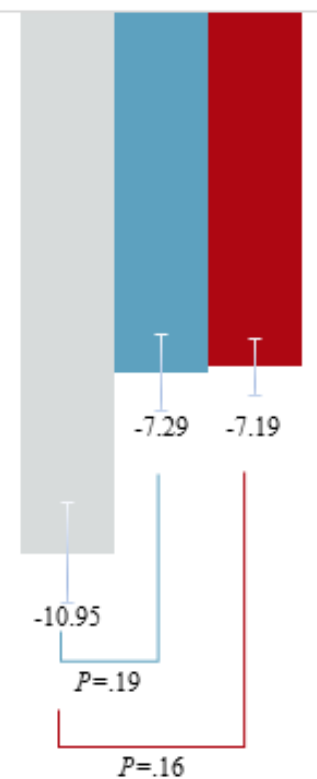


Figure 4. Body weight changes from the baseline across the 3 groups at each time point. AO: app-only; APC: app with personalized coaching; CO: control, SBP: systolic blood pressure.

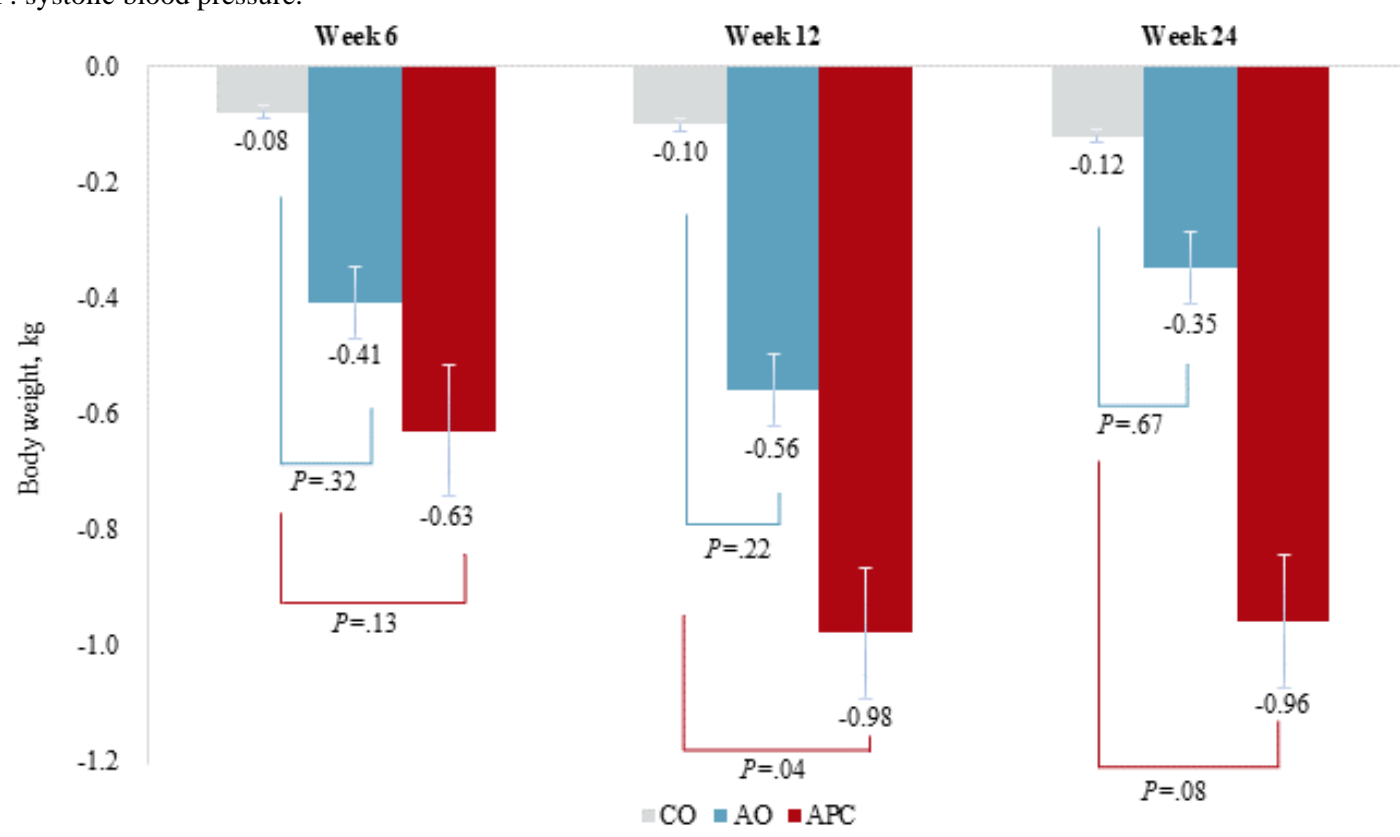

Figure 5. Body fat mass changes from the baseline across the 3 groups at each time point. AO: app-only; APC: app with personalized coaching; CO: control.

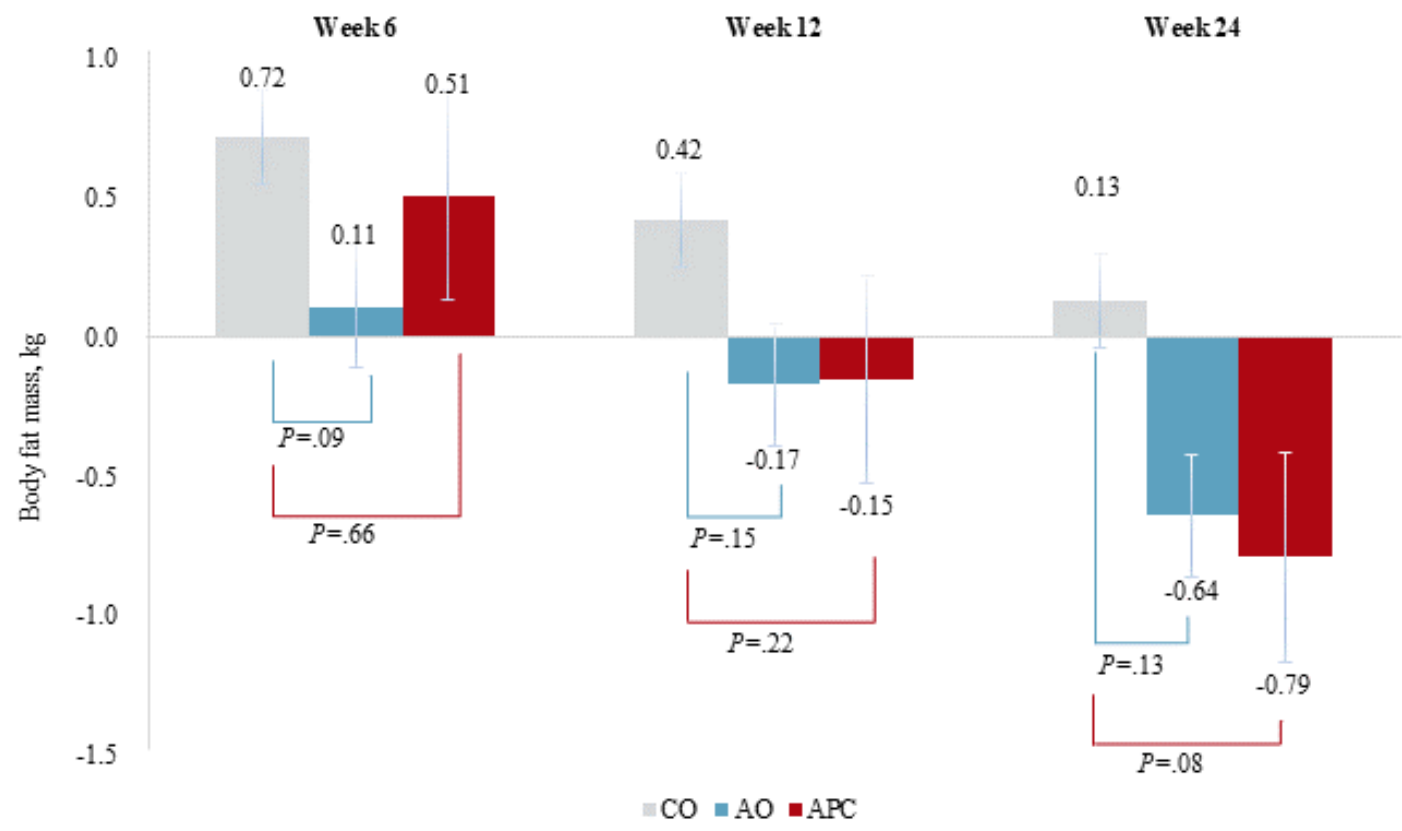

\section{Linear Mixed Model}

Neither the app only (control versus app only at week 24: $\beta=2.93$, SE 2.42, $P=.23$ ) nor the app and additional personalized feedback feature (control versus app with personalized coaching at week 24: $\beta=3.18$, SE 2.42, $P=.19$ ) significantly contributed to systolic blood pressure reduction at any of the follow-up examinations (Multimedia Appendix 1). In regard to secondary outcomes, statistically significant effects were observed for body weight and body fat. Compared to the participants in the control group, those in the app with personalized coaching group lost more body weight at week $12(\beta=-0.93$, SE $0.43 ; P=.03)$ and at week $24(\beta=-0.87$, SE $0.42 ; P=.04)$. This translated to an average difference of $0.14 \mathrm{~kg}$ of body weight loss per month between participants in the control group and participants in the app with personalized coaching group. In parallel, the app with personalized coaching group lost more body fat than the control group did at week 24 ( $\beta=-0.95$, SE $0.46, P=.04)$. This was equivalent to participants in the app with personalized coaching group losing an average of $0.19 \mathrm{~kg}$ more body fat per month than those in the control group. The app with personalized coaching group showed significant HOMA-IR level reductions at week $12(\beta=-0.55, \mathrm{SE} 0.27, P=.04)$. However, separate examination by fasting glucose level did not show significance $(P=.80)$ and the HOMA-IR reductions diminished by week 24 ( $\beta=-0.22$, SE $0.26, P=.40$ ). Otherwise, there were no significant effects of the app on diastolic blood pressure (app only: $P=.83$; app with personalized coaching group: $P=.47$ ), waist 
circumference (app only: $P=.32$; app with personalized coaching: $P=.12$ ), triglyceride (app only: $P=.70$; app with personalized coaching: $P=.23$ ), HDL cholesterol (app only: $P=.14$; app with personalized coaching: $P=.91$ ), or fasting blood glucose level (app only: $P=.38$; app with personalized coaching: $P=.84$; Multimedia Appendix 1).

\section{Discussion}

\section{Principal Findings}

Mobile-based behavioral health interventions that permit real-time data collection and sharing are increasingly commonplace, enabling researchers to assess multiple health behaviors in various contexts. At the same time, they prompt users to be more self-aware and to modify their own health behavior. In this context, the goal behind this study was to assess the real-world effectiveness of a smartphone-based self-monitoring health management app in a community-dwelling population of individuals without diagnosis or treatment of metabolic disorders. App usage showed differential effects on metabolic parameters at different time points. Overall, the primary outcome of systolic blood pressure followed a decreasing trend from the baseline yet did not change notably between the 3 groups at any follow-up examinations. Instead, the simultaneous diet/exercise logging and lifestyle coaching yielded relatively greater body weight reduction, specifically via body fat mass reduction. These effects were attenuated yet sustained at 6 month.

Despite no significant systolic blood pressure improvement in this study, previous studies [12-14,22-24] have identified the utility of mobile apps in lowering blood pressure through self-monitoring and information delivery services. In several randomized controlled trials [12-14,22-24] conducted in obesity clinic settings, text messages or emails for antihypertensive medication adherence, tailored guidance on salt intake, smoking cessation, and physical activity interventions reported significant differences in blood pressure reduction compared to usual care. However, unlike our study in which community-dwelling individuals without overt metabolic syndrome participated, similar studies $[7,21,25]$ have been primarily performed on patients based on completers' analysis rather than intention-to-treat. Considering varied levels of initiative for health management often used as a proxy for adherence and attrition, our results indicate that effectiveness of these self-care apps may considerably differ by study population and analytical methods.

Moreover, Liu and colleagues' [25] examination of internet-based counseling interventions among patients with elevated blood pressure indicated that e-counseling interventions significantly reduced daytime systolic blood pressure by 3.8 $\mathrm{mmHg}$ (95\% CI -5.63 to -2.06$)$, with greater reductions from more sustained interventions. Since our study relied on a single-occasion blood pressure measurement in the examination setting, the results may potentially be distorted by whitecoat or masked hypertension.

Furthermore, a significant limitation of accumulated evidence is that many studies [7] were conducted for durations less than
6 months. Given that hypertension is a chronic condition that requires long-term pharmacological treatment and lifestyle modification, prolonged observation should be allotted for more accurate assessment of intervention adherence and subsequent blood pressure changes.

In our study, body weight reduction, via body fat mass loss, was the most evident improvement from app usage, as indicated by similar trials [26,27]. Among overweight adults, daily transmitted personalized multimedia message services providing weight control materials have proven to induce greater weight loss $(-1.97 \mathrm{~kg}$ difference, $95 \% \mathrm{CI}-0.34$ to $-3.60 \mathrm{~kg})$ than the non-receiving group [26]. A systematic review [25] consistently identified the association between self-monitoring or online obesity treatment programs and body size reduction via targeted advice on reduced energy intake, increased physical activity, and social support. Often, the studies incorporated the use of structured regimen, regular self-monitoring, circumstantially appropriate feedback, prompt communication, and social support [27]. Abraham et al [28] classified such intervention content, including information-motivation-behavior skills model (ie, providing information about behavior-health link), social-cognitive theory (ie, prompting barrier identification, general encouragement), control therapy (ie, self-monitoring, interactive feedback), relapse prevention and behavior sustenance, and more. Although the extent and nature of the interventions varied across studies, these elements commonly found in mobile technology interventions, altogether, appear crucial in driving greater changes.

Our study results indicated that the 6-month usage of the mobile app did not substantially improve insulin sensitivity. Yet, an analogous meta-analysis [29] demonstrated that comprehensive lifestyle modification delivered through a mobile app lowered hemoglobin $A_{1 c}$ level by an average of $0.5 \%$ over 6 months of follow-up. Interestingly, this decrease was not accompanied by concurrent improvements in other diabetes risk factors, such as blood pressure, cholesterol levels, or adiposity [29]. However, considering that hemoglobin $\mathrm{A}_{1 \mathrm{c}}$ reflects long-term fluctuations of glycemic control (unlike fasting glucose level used in our study), Liang et al [29] raised concerns regarding insufficient follow-up period. Moreover, since these trials were conducted on persons diagnosed with diabetes of varying subtypes and adherence to antihyperglycemic treatments, it would have been challenging to isolate the effectiveness of the mobile app intervention from changes in treatment behavior. Therefore, the different representation of glycemic state and participant characteristics warrant caution in assuming or denying the attribution of these technology-based intervention to other nonspecific benefits.

Over the span of 6 months, our participants did not show significantly different changes in triglyceride and HDL cholesterol levels across the 3 groups. Yet, Park and Kim [30] showed that, among gynecology and family medicine outpatients, the use of web-based diet and exercise diaries and the specialists' weekly lifestyle modification recommendations improved total $(-12.9 \mathrm{mg} / \mathrm{dL})$ and low-density lipoprotein $(-11.3$ $\mathrm{mg} / \mathrm{dL}$ ) cholesterol levels after 12 weeks. Again, it is hard to extrapolate from clinic setting population in which individuals 
may already be utilizing health management app prior to trial entrance or may be taking medications (ie, statins) that distort the true effects of the interventions.

The greatest novelty of our study lies in its evaluation of the mobile app's effectiveness via intention-to-treat approach in nonpatient population without the concern of confounding by pharmacological treatment. Considering that the general population consists of individuals with wide-ranging levels of digital literacy and utility, willingness for lifestyle modification, and physiological and socioeconomic backgrounds, our study portrays the effectiveness of mobile-based health management app in real-world setting. Furthermore, the assessor-blinding enabled true compliance assessment in the sample regardless of individual preference for or competency with the app, thereby evaluating the app's real-world potential. In addition, because we assessed the changes in metabolic parameters repeatedly over a considerable time horizon, our results reflect the long-term trajectory of the metabolic indices, empirically. Lastly, as a 3-arm parallel design with concurrent control, we were able to discriminate whether the self-logging of diet and exercise was sufficient to induce improvements in metabolic parameter or whether additional personalized coaching was the critical element in shaping healthy behavior.

Nonetheless, several limitations warrant cautious interpretation of our study findings. During the screening test, we faced an unexpectedly greater number of participants who did not satisfy the inclusion criteria, and thus, we were unable to reach the goal sample size. During the study period, because the participants had varying attendance to each of the follow-up examination, there may have been residual heterogeneity among participants who attended follow-up examinations none, once, twice, or all follow-up examinations. Yet, those lost to follow-up were demographically similar to those who participated in the study with the exception of current smoking status. If any effects were present, we expect such attrition to have an effect on the study results toward the null. Moreover, because the participants were selected from an already-established cohort, higher self-efficacy and proficiency may have been present. Therefore, the results may not be entirely generalizable to the general population. However, the original cohort study was purely observational; thus, our findings still represent individuals typically seeking health management without referral to physician nor pharmacological treatment. Furthermore, similar literature has suggested that baseline self-efficacy appraisals may not be entirely pertinent to practical skills or opportunities to sustain life changes [15]. In this context, we expect comparable initiatives for lifestyle modification between our study and the general population. Lastly, considering that blood pressure, body size, glycemic index, and lipid indices are reflective of chronic states, our 6-month study period may have been insufficient to witness changes. Future studies with a larger sample size and a prolonged study period may better assess the effectiveness of lifestyle modification mobile apps on the long-term trajectory of metabolic indices.

\section{Conclusions}

Among the community-dwelling adults with moderate metabolic abnormalities without diagnosis or treatment for disorders, we examined the effect of a smartphone-based app on changes in metabolic parameters. By the end of the 6-month study period, no changes to systolic blood pressure were significant for participants who utilized both diet/exercise logging and personalized coaching compared to logging-only or nonusing groups. Instead, the self-logging and lifestyle coaching yielded greater body weight reduction via body fat mass loss. The research investigating mobile health management interventions that confer accessible and cost-effective benefits remains in its infancy, especially in general populations. Future studies focusing on comparative effectiveness using alternative study designs and on populations of various health, socioeconomic, and cultural backgrounds are needed to integrate these apps in everyday lives and clinic practice.

\section{Acknowledgments}

This study was supported by a grant from the Korean Health Technology R \& D Project, Ministry of Health \& Welfare, Korea (HI13C0715). The Ministry of Health \& Welfare did not have any role in the writing of the manuscript nor in the decision to submit the manuscript for publication. The authors have not been paid to write this article by any pharmaceutical company or any other agency. The corresponding author had full access to all the data in the study and had final responsibility for the decision to submit for publication.

\section{Authors' Contributions}

JHL, J-SS, HY, and HCK conceived and designed the trial. J-SS and HCK developed the education program. SMJC, JHL, J-SS, HY, YWJ, and HCK helped perform the study. SMJC, JHL, J-SS, HY, YWJ, and HCK acquired the data. SMJC, JHL, and HCK analyzed and interpreted the data. SMJC and JHL drafted the manuscripts. SMJC, JHL, and HCK critically revised the manuscript. SMJC and JHL performed the statistical analysis. J-SS and HCK supervised the study. All authors approved the final version of the manuscript.

\section{Conflicts of Interest}

None declared.

\section{Multimedia Appendix 1}

Supplementary tables. 
[DOCX File , 44 KB-Multimedia Appendix 1]

\section{Multimedia Appendix 2}

Diastolic blood pressure changes from the baseline across the 3 groups at each time point. AO: app-only; APC: app with personalized coaching; CO: control.

[PNG File, 12 KB-Multimedia Appendix 2]

\section{Multimedia Appendix 3}

Waist circumference changes from the baseline across the 3 groups at each time point. AO: app-only; APC: app with personalized coaching; CO: control.

[PNG File, 10 KB-Multimedia Appendix 3]

\section{Multimedia Appendix 4}

Fasting glucose level changes from the baseline across the 3 groups at each time point. AO: app-only; APC: app with personalized coaching; CO: control.

[PNG File, $11 \mathrm{~KB}-$ Multimedia Appendix 4]

\section{Multimedia Appendix 5}

Homeostatic model of assessment of insulin resistance changes from the baseline across the 3 groups at each time point. AO: app-only; APC: app with personalized coaching; CO: control.

[PNG File, $11 \mathrm{~KB}-\underline{\text { Multimedia Appendix 5] }}$

\section{Multimedia Appendix 6}

Triglyceride level changes from the baseline across the 3 groups at each time point. AO: app-only; APC: app with personalized coaching; CO: control.

[PNG File, 10 KB-Multimedia Appendix 6]

\section{Multimedia Appendix 7}

High-density lipoprotein cholesterol level changes from the baseline across the 3 groups at each time point. AO: app-only; APC: app with personalized coaching; CO: control.

[PNG File, 15 KB-Multimedia Appendix 7]

\section{Multimedia Appendix 8}

CONSORT-eHEALTH checklist (V 1.6.1).

[PDF File (Adobe PDF File), 392 KB-Multimedia Appendix 8]

\section{References}

1. Wilson PWF, D'Agostino RB, Parise H, Sullivan L, Meigs JB. Metabolic syndrome as a precursor of cardiovascular disease and type 2 diabetes mellitus. Circulation 2005 Nov 15;112(20):3066-3072 [FREE Full text] [doi: 10.1161/CIRCULATIONAHA.105.539528] [Medline: 16275870]

2. Mottillo S, Filion KB, Genest J, Joseph L, Pilote L, Poirier P, et al. The Metabolic Syndrome and Cardiovascular Risk. Journal of the American College of Cardiology 2010 Sep;56(14):1113-1132. [doi: 10.1016/j.jacc.2010.05.034]

3. Kang S, Kim S, Cho JH, Yoon C, Hwang S, Lee H, et al. Prevalence, Awareness, Treatment, and Control of Hypertension in Korea. Sci Rep 2019 Jul 29;9(1):10970 [FREE Full text] [doi: 10.1038/s41598-019-46965-4] [Medline: 31358791]

4. Kim B, Won JC, Lee JH, Kim H, Park JH, Ha KH, et al. Diabetes Fact Sheets in Korea, 2018: An Appraisal of Current Status. Diabetes Metab J 2019;43(4):487. [doi: 10.4093/dmj.2019.0067]

5. Jeong JS, Kwon HS. Prevalence and Clinical Characteristics of Dyslipidemia in Koreans. Endocrinol Metab (Seoul) 2017 Mar;32(1):30-35 [FREE Full text] [doi: 10.3803/EnM.2017.32.1.30] [Medline: 28345314]

6. Garrett P, Brown CA, Hart-Hester S, Hamadain E, Dixon C, Pierce W, et al. Identifying barriers to the adoption of new technology in rural hospitals: a case report. Perspect Health Inf Manag 2006 Oct 25;3:9 [FREE Full text] [Medline: 18066367]

7. Mosa ASM, Yoo I, Sheets L. A systematic review of healthcare applications for smartphones. BMC Med Inform Decis Mak 2012;12:67 [FREE Full text] [doi: 10.1186/1472-6947-12-67] [Medline: 22781312] 
8. Boulos MNK, Brewer AC, Karimkhani C, Buller DB, Dellavalle RP. Mobile medical and health apps: state of the art, concerns, regulatory control and certification. Online J Public Health Inform 2014;5(3):229 [FREE Full text] [doi: 10.5210/ojphi.v5i3.4814] [Medline: 24683442]

9. Burke LE, Ma J, Azar KMJ, Bennett GG, Peterson ED, Zheng Y, American. Current Science on Consumer Use of Mobile Health for Cardiovascular Disease Prevention: A Scientific Statement From the American Heart Association. Circulation 2015 Sep 22;132(12):1157-1213. [doi: 10.1161/CIR.0000000000000232] [Medline: 26271892]

10. Pandey A, Krumme A, Patel T, Choudhry N. The Impact of Text Messaging on Medication Adherence and Exercise Among Postmyocardial Infarction Patients: Randomized Controlled Pilot Trial. JMIR Mhealth Uhealth 2017 Aug 03;5(8):e110 [FREE Full text] [doi: 10.2196/mhealth.7144] [Medline: 28778843]

11. Haapala I, Barengo NC, Biggs S, Surakka L, Manninen P. Weight loss by mobile phone: a 1-year effectiveness study. Public Health Nutr 2009 Dec;12(12):2382-2391. [doi: 10.1017/S1368980009005230] [Medline: 19323865]

12. Cottrell E, Chambers R, O'Connell P. Using simple telehealth in primary care to reduce blood pressure: a service evaluation. BMJ Open 2012;2(6) [FREE Full text] [doi: 10.1136/bmjopen-2012-001391] [Medline: 23117563]

13. Kang S, Kim S, Cho JH, Yoon C, Hwang S, Lee H, et al. Prevalence, Awareness, Treatment, and Control of Hypertension in Korea. Sci Rep 2019 Jul 29;9(1):10970 [FREE Full text] [doi: 10.1038/s41598-019-46965-4] [Medline: 31358791]

14. Logan AG, Irvine MJ, McIsaac WJ, Tisler A, Rossos PG, Easty A, et al. Effect of home blood pressure telemonitoring with self-care support on uncontrolled systolic hypertension in diabetics. Hypertension 2012 Jul;60(1):51-57 [FREE Full text] [doi: 10.1161/HYPERTENSIONAHA.111.188409] [Medline: 22615116]

15. Whelton PK, Carey RM, Aronow WS, Casey DE, Collins KJ, Dennison HC, et al. 2017 ACC/AHA/AAPA/ABC/ACPM/AGS/APhA/ASH/ASPC/NMA/PCNA Guideline for the Prevention, Detection, Evaluation, and Management of High Blood Pressure in Adults: A Report of the American College of Cardiology/American Heart Association Task Force on Clinical Practice Guidelines. Hypertension 2017 Nov 13:e13-e115. [doi: 10.1161/HYP.0000000000000065] [Medline: 29133356]

16. Williams B, Mancia G, Spiering W, Agabiti Rosei E, Azizi M, Burnier M, ESC Scientific Document Group. 2018 ESC/ESH Guidelines for the management of arterial hypertension. Eur Heart J 2018 Sep 01;39(33):3021-3104. [doi: 10.1093/eurheartj/ehy339] [Medline: $\underline{\text { 30165516] }}$

17. Shim J, Song BM, Lee JH, Lee SW, Park JH, Choi DP, et al. Cardiovascular and Metabolic Diseases Etiology Research Center (CMERC) cohort: study protocol and results of the first 3 years of enrollment. Epidemiol Health 2017;39:e2017016 [FREE Full text] [doi: 10.4178/epih.e2017016] [Medline: 28395401]

18. National Cholesterol Education Program Expert Panel. Third Report of the National Cholesterol Education Program (NCEP) Expert Panel on Detection, Evaluation, and Treatment of High Blood Cholesterol in Adults (Adult Treatment Panel III) Final Report. Circulation 2002 Dec 17;106(25):3143-3143. [doi: 10.1161/circ.106.25.3143]

19. Chun MY. Validity and Reliability of Korean Version of International Physical Activity Questionnaire Short Form in the Elderly. Korean J Fam Med 2012;33(3):144. [doi: 10.4082/kjfm.2012.33.3.144]

20. Twisk J, Bosman L, Hoekstra T, Rijnhart J, Welten M, Heymans M. Different ways to estimate treatment effects in randomised controlled trials. Contemp Clin Trials Commun 2018 Jun;10:80-85 [FREE Full text] [doi: 10.1016/j.conctc.2018.03.008] [Medline: 29696162]

21. Schippers M, Adam PCG, Smolenski DJ, Wong HTH, de Wit JBF. A meta-analysis of overall effects of weight loss interventions delivered via mobile phones and effect size differences according to delivery mode, personal contact, and intervention intensity and duration. Obes Rev 2017 Apr;18(4):450-459. [doi: 10.1111/obr.12492] [Medline: 28187246]

22. Madsen LB, Kirkegaard P, Pedersen EB. Blood pressure control during telemonitoring of home blood pressure. A randomized controlled trial during 6 months. Blood Press 2008;17(2):78-86. [doi: 10.1080/08037050801915468] [Medline: 18568696]

23. Nolan RP, Liu S, Shoemaker JK, Hachinski V, Lynn H, Mikulis DJ, et al. Therapeutic benefit of internet-based lifestyle counselling for hypertension. Can J Cardiol 2012 May;28(3):390-396. [doi: 10.1016/j.cjca.2012.02.012] [Medline: 22498181]

24. Piette JD, Datwani H, Gaudioso S, Foster SM, Westphal J, Perry W, et al. Hypertension management using mobile technology and home blood pressure monitoring: results of a randomized trial in two low/middle-income countries. Telemed J E Health 2012 Oct;18(8):613-620 [FREE Full text] [doi: 10.1089/tmj.2011.0271] [Medline: 23061642]

25. Liu S, Dunford SD, Leung YW, Brooks D, Thomas SG, Eysenbach G, et al. Reducing blood pressure with Internet-based interventions: a meta-analysis. Can J Cardiol 2013 May;29(5):613-621. [doi: 10.1016/j.cjca.2013.02.007] [Medline: 23618507]

26. Patrick K, Raab F, Adams MA, Dillon L, Zabinski M, Rock CL, et al. A text message-based intervention for weight loss: randomized controlled trial. J Med Internet Res 2009;11(1):e1 [FREE Full text] [doi: 10.2196/jmir.1100] [Medline: 19141433]

27. Khaylis A, Yiaslas T, Bergstrom J, Gore-Felton C. A review of efficacious technology-based weight-loss interventions: five key components. Telemed J E Health 2010 Nov;16(9):931-938 [FREE Full text] [doi: 10.1089/tmj.2010.0065] [Medline: $\underline{21091286}$

28. Abraham C, Michie S. A taxonomy of behavior change techniques used in interventions. Health Psychol 2008 May;27(3):379-387. [doi: 10.1037/0278-6133.27.3.379] [Medline: 18624603] 
29. Liang X, Wang Q, Yang X, Cao J, Chen J, Mo X, et al. Effect of mobile phone intervention for diabetes on glycaemic control: a meta-analysis. Diabet Med 2011 Apr;28(4):455-463. [doi: 10.1111/j.1464-5491.2010.03180.x] [Medline: 21392066]

30. Park M, Kim H. Evaluation of mobile phone and Internet intervention on waist circumference and blood pressure in post-menopausal women with abdominal obesity. Int J Med Inform 2012 Jun;81(6):388-394. [doi: 10.1016/j.ijmedinf.2011.12.011] [Medline: 22265810]

\section{Abbreviations \\ CMERC: Cardiovascular and Metabolic Diseases Etiology Research Center \\ HDL: high-density lipoprotein \\ HOMA-IR: homeostatic model of assessment of insulin resistance}

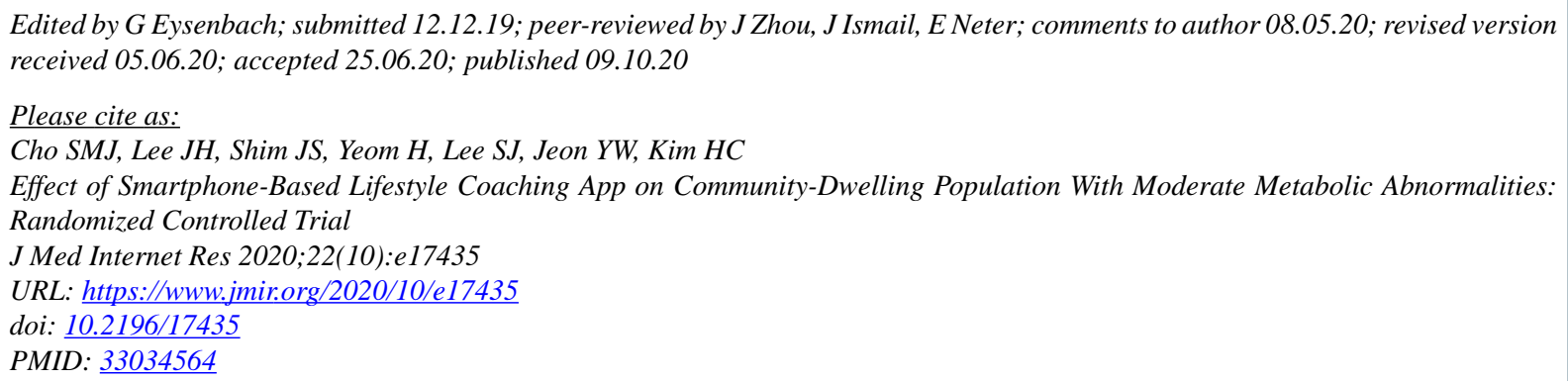

(CSo Mi Jemma Cho, Jung Hyun Lee, Jee-Seon Shim, Hyungseon Yeom, Su Jin Lee, Yong Woo Jeon, Hyeon Chang Kim. Originally published in the Journal of Medical Internet Research (http://www.jmir.org), 09.10.2020. This is an open-access article distributed under the terms of the Creative Commons Attribution License (https://creativecommons.org/licenses/by/4.0/), which permits unrestricted use, distribution, and reproduction in any medium, provided the original work, first published in the Journal of Medical Internet Research, is properly cited. The complete bibliographic information, a link to the original publication on http://www.jmir.org/, as well as this copyright and license information must be included. 